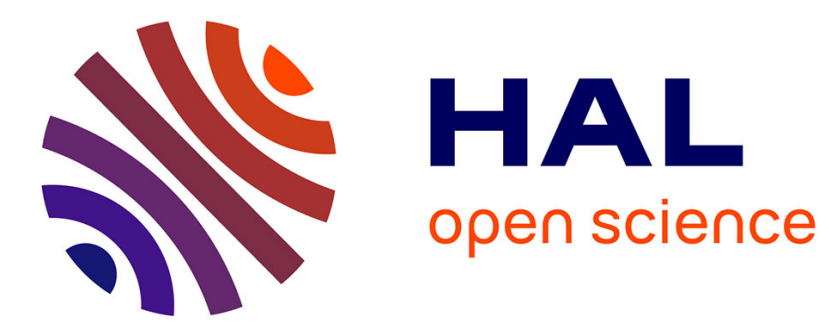

\title{
L'amitié, les amis, leur histoire. Représentations et récits
}

Claire Bidart

\section{To cite this version:}

Claire Bidart. L'amitié, les amis, leur histoire. Représentations et récits. Sociétés contemporaines, 1991, 5, pp.21-42. halshs-00132989

\section{HAL Id: halshs-00132989 \\ https://shs.hal.science/halshs-00132989}

Submitted on 6 Mar 2007

HAL is a multi-disciplinary open access archive for the deposit and dissemination of scientific research documents, whether they are published or not. The documents may come from teaching and research institutions in France or abroad, or from public or private research centers.
L'archive ouverte pluridisciplinaire $\mathbf{H A L}$, est destinée au dépôt et à la diffusion de documents scientifiques de niveau recherche, publiés ou non, émanant des établissements d'enseignement et de recherche français ou étrangers, des laboratoires publics ou privés. 
Claire Bidart

CERCOM

EHESS-CNRS

Vieille Charité

2 rue de la Charité

13002 Marseille

\section{L'amitié, les amis, leur histoire}

\section{Représentations et récits}

Résumé: La prise en considération de la dimension subjective des relations interpersonnelles, de la façon dont les acteurs les perçoivent et les décrivent, doit s'inscrire dans la démarche de compréhension de ces relations mise en oeuvre par les sciences sociales et les études de réseaux en particulier. L'analyse d'une série d'entretiens nous permet de distinguer trois niveaux de discours sur l'amitié: le niveau d'une définition générale; le niveau des représentations de relations réelles; et le niveau du récit de l'élection, au moment de la fondation de la relation. On compare, pour différentes catégories sociales, l'agencement de ces niveaux. On peut constater que les situations dramatiques, exceptionnelles, à l'écart des cadres sociaux ordinaires, président souvent à la fondation des relations d'amitié.

Abstract: Consideration of the subjective dimension of interpersonal relationships, of the ways individuals perceive and describe them, must be integrated into research endeavours about those relationships implemented by the social sciences and network studies specifically. Concerning friendship, I distinguish here three levels of discourse collected from interviews: the level of the general definitions of friendships; the level of the representations of individuals' active relationships; and the level of the account of election, at the moment of friendship founding. I compare, for different social categories, the organization of these levels. One may establish that emergency situations, generally apart from ordinary social settings, are often at the foundation of friendship relations.

L'attention portée à la sociabilité comme forme constitutive de la vie sociale a été initiée par les travaux de G. Simmel (1917, trad. 1981). Il est beaucoup plus difficile de situer l'émergence de l'amitié comme objet. Divers champs disciplinaires connexes l'ont traitée au sein de leurs études sur la sociabilité, mais sans la distinguer véritablement. On peut même dire que le plus grand flou règne généralement. Les Anglo-saxons utilisent le terme "friendship" pour désigner des relations de natures très diverses (et en général non précisées), qui n'excluent pas, contrairement au terme français, les rapports avec des parents. Si des précisions telles que "close friends" et "non-kin" permettent de se rapprocher du domaine de définition du mot français, peu d'auteurs finalement ont cherché à préciser le sens et la spécificité de l'amitié. Différents types de travaux, issus de lignées théoriques et méthodologiques diverses, ont contribué, chacun de leur côté, à donner un éclairage spécifique sur l'amitié. Peut-être serait-il temps de mettre en oeuvre leurs complémentarités.

Les études de réseaux trouvent leur origine avec les travaux sociométriques de J.L. Moreno (1970, lè édition 1934). On en a surtout retenu la dimension "technique". Et de fait, les études de réseaux seront définies, pendant longtemps, par leur caractère spécifiquement méthodologique. En France, les études de réseaux ont débuté avec la formalisation mathématique (Flament, 1965). R.K. Merton et P.F. Lazarsfeld, 
en signalant la ligne de séparation entre l'approche substantive et l'approche méthodologique $\left({ }^{1}\right)$, ne soupçonnaient peut-être pas à quel point elle serait encore pertinente longtemps après. Les études de réseaux se sont longtemps situées du côté de la forme, de la méthodologie. Certains s'inquiètent encore aujourd'hui de ce qui pourrait apparaître comme un "vide théorique", voire une absence de questionnement spécifique $\left(^{2}\right)$.

L'objet des études de réseaux, tout en restant dominé par les questions méthodologiques, a évolué depuis les années soixante-dix qui ont marqué leur véritable démarrage.

Cet objet a été tout d'abord constitué autour des relations interindividuelles comme unité de base; il s'agissait de dresser la liste des contacts personnels entretenus par un individu. Mais cette collection isolait, déconnectait arbitrairement les relations les unes des autres. Les chercheurs ont considéré alors le réseau comme un système, dont ils ont étudié la structure en tant que réalité douée d'une pertinence globale, dont la signification dépasse la somme des relations diadiques. Ils ont cherché aussi à rattacher ces réseaux aux structures sociales plus larges, en reconstruisant le rapport entre les liens interpersonnels et les groupes sociaux (que les études de réseaux avaient primitivement déconstruits, à partir notamment de la critique des "community studies"; cf Wellman \& Leighton 1981); certains ont fait appel notamment au concept de cercles sociaux (Degenne, 1983), qui réintroduit dans le réseau la notion de groupe d'appartenance.

En ouvrant leur problématique, mais aussi en étendant leur portée théorique, c'est donc une dimension structurale que tendent à prendre aujourd'hui les études de réseaux (Burt, 1982, Marsden \& Lin, 1982, Wellman, 1987, 1988).

Les mouvements de cette quête théorique peuvent être un signe de dynamisme, mais aussi de fragilité de cette approche, "méthode puissante à la recherche d'une théorie unifiante" (Ferrand, 1986).

D'autres disciplines ont, de leur côté, traité de l'amitié.

C'est à partir de l'étude des relations de parenté, et toujours en lien étroit avec elles, que les anthropologues ont exploré les relations d'amitié. Ils ont rarement traité ces dernières de manière autonome. Il est vrai que sur leurs terrains traditionnels, l'amitié est parfois institutionalisée, ritualisée comme les rapports de parenté (Pitt-Rivers, 1963, 1968, Eisenstadt, 1956). Il est de fait intéressant, pour la compréhension d'un concept, de le confronter aux catégories qui lui sont adjacentes. On note d'ailleurs que dans nos sociétés occidentales, l'ami et le frère sont souvent mis en rapport, dans le sens commun. Dans la ligne de la tradition en anthropologie de la parenté, l'amitié a souvent été étudiée plus dans ses règles que dans ses manifestations et leurs variations. C'est le cas en particulier des études qui dressent de vastes tableaux comparatifs entre des sociétés très éloignées (Cohen, 1961).

Les anthropologues ont aussi fortement mis l'accent sur l'influence du contexte dans lequel s'établissent les relations. Les travaux de l'école de Chicago ont marqué un tournant décisif en ce domaine (Park \& Burguess, 1925, Wirth, 1938, Hugues, 1958 ... voir aussi Hannerz, 1983, Grafmeyer \& Joseph, 1979, Sennett, 1980 a \& b, Becker, 1985). Les quartiers des grandes villes, les banlieues, ont été l'objet d'investigations mettant en lumière les interactions entre milieu local, relations interpersonnelles et socialisation (Young et Willmott, 1983, Firth, Hubert \& Forge, 1969, Bell 1968, Adams, 1968).

Certains auteurs ont établi un lien direct avec les études de réseaux, en s'attachant à montrer les

\footnotetext{
1 A line of division "is that between sociological theories which, comprised of concepts and propositions about social behavior, are substantive in character, and methodology which, comprised of statements about the logic of inquiry, is necessarily formal, rather than substantive, in character. (...) The one is primarily concerned with the "what" of sociological inquiry; the other, with the "how"." (Lazarsfeld \& Merton, 1954, pp.18-19).

2 "Some reject it (network analysis) as mere methodology lacking due regard for substantive issues" (Wellman, 1983, p.155)

"Network analysis might be described as the product of an invisible college of tool-makers searching for a nail to pound" (Rogers, 1987, p. 295)
} 
interactions entre le réseau individuel et la relation de couple (Bott, 1957, Babchuk, 1965, Babchuk \& Bates, 1963).

Proche de l'interactionnisme symbolique, Paine $(1969,1970)$ s'est attaché à explorer l'amitié telle que la définissent les acteurs eux-mêmes, les valeurs qu'ils y investissent, et les liens avec les institutions sociales.

La psychologie sociale a apporté une contribution très importante à la définition idiosyncratique de l'amitié. Elle étudie et met en rapport diverses dimensions de la relation amicale, en s'attachant à explorer les interactions entre individu et société. L'influence des milieux, des "climats sociaux locaux" (Lazarsfeld \& Merton, 1954), de la "vicinité" (Maisonneuve, 1966), ainsi que d'autres variables plus individuelles (principalement l'âge, le sexe, plus rarement le "niveau social" ou la profession), sont étudiées en tant que déterminants de la construction des affinités. La notion d'homophilie $\left(^{3}\right)$ est centrale en ce qu'elle tend à rendre compte des critères sociaux de "sélection" des amis. La psychologie sociale étudie aussi le rapport aux normes, aux règles sociales de la relation d'amitié (Argyle, 1986, Suttles, 1970), aux valeurs aussi (Davis \& Todd, 1982). Elle mesure les variations du lien amical au cours du cycle de vie (Dickens \& Perlman, 1981), son rôle dans la socialisation et le développement de la personnalité (Duck, 1988, Derlega \& Winstead, 1986, Furnham, 1989). Considérant l'amitié comme un processus relationnel, elle explore la diversité des liens (Hays, 1988), leurs différentes phases et transformations, leurs modalités d'évolution (Perlman \& Duck, 1987, Duck \& Gilmour, 1981, Duck, 1982). Les perceptions de la relation par les acteurs sont aussi parfois étudiées (Serafica, 1983), ainsi que les interactions cognitives entre les partenaires (Maisonneuve, 1966). Les travaux sur les "effets" individuels des relations d'amitié, ou de la solitude, rejoignent les études de réseaux qui sont aujourd'hui de plus en plus nombreuses à étudier les conséquences de la structuration des réseaux individuels sur le bien-être, l'insertion sociale, et les maladies mentales.

Certains travaux historiques apportent également leur pierre à l'édifice (Agulhon, 1977, Ariès \& Duby, 1987), en particulier ceux qui nous donnent un aperçu de l'histoire des idées sur l'amitié (Aymard, 1986, Silver, 1989), notamment à travers la littérature et les grands textes fondateurs grecs et judéo-chrétiens (Fraisse, 1974, Easterling, 1989, Clark, 1989).

Il est parfois artificiel de séparer ainsi les disciplines. L'école de Chicago a eu une influence qui dépasse bien largement le cercle de l'anthropologie. Si les études de réseaux s'attachent maintenant à explorer plus avant la façon dont les contextes déterminent les relations (Fischer, 1982, Huckfeldt, 1983, Feld, 1984); si elles mesurent les effets de la structuration du réseau individuel sur les ressources mobilisables (Granovetter, 1973, 1974, 1982, Wellman, 1985, Reichman, 1987), sur l'aide psychologique (Wellman, 1981, Wellman \& Berkowitz, 1988, Ormel, Van Tilburg \& Van Sonderen, 1989, Burt, 1987, Willmott, 1987), voire sur des perceptions sociales comme le sentiment d'insécurité (Lagrange \& Roché, 1988); et si elles cherchent à approfondir la définition du contenu des relations (Marsden \& Campbell, 1984, Ferrand, 1985, 1989, Bidart, 1988b), ce n'est sans doute pas sans lien avec les travaux de l'anthropologie et de la psychologie sociale.

Il reste que si ces liens existent, ils sont encore trop peu développés. La construction des objets dans les sciences sociales, la délimitation de leurs champs n'ont de fait pas attribué à l'amitié une place assignée dans une discipline spécifique. Et c'est sans doute une bonne chose, la pluralité des points de vue ne pouvant qu'en enrichir la compréhension, à condition d'établir et de multiplier les échanges entre disciplines. S'ils veulent néanmoins construire et autonomiser cet objet, les sociologues devront d'autant plus travailler avec leurs "voisins" anthropologues, psycho-sociologues, historiens, dans une

\footnotetext{
3 Merton \& Lazarsfeld (1954) la définissent ainsi: "a tendancy for friendships to form between those who are alike in some designated respect" (p.23). Cette notion est calquée sur celle d'homogamie.
} 
interdisciplinarité qui est par ailleurs tout à fait de mise aujourd'hui. L'étude de l'amitié devra être, à mon sens, considérée comme un sous-ensemble des études sur la sociabilité, un sous-ensemble à la fois distinct et complémentaire de ces dernières.

Rares sont les auteurs qui, comme G. Allan (1979), prennent l'amitié pour objet central $\left({ }^{4}\right)$. Elle est généralement plutôt traitée comme une forme de sociabilité, non particularisée, non spécifiée par rapport à cette dernière. L'attention sociologique s'est surtout portée sur les différences entre classes populaires et classes moyennes. Il s'agit dans la plupart des cas de tester la pertinence de diverses variables sur les relations interpersonnelles. Certains travaux mettent également en lumière la pregnance des "milieux sociaux", dans une problématique qui tente de réconcilier la monographie avec le comparatisme (Paradeise, 1980, Bidart, 1988a). Ils cherchent en particulier à comparer les degrés et les formes de leur impact sur les relations interpersonnelles, selon que ces milieux sont plus ou moins "institutionalisés": les conditions d'émergence et de fonctionnement des liens interpersonnels ne sont pas les mêmes dans une entreprise où les rapports sont "a priori" formalisés, hiérarchisés, où linteraction est instituée par la nécessité de réaliser une tâche en commun, et dans un quartier où l'évitement est possible, et où "rien n'est joué a priori" dans les rapports sociaux. (Bidart, 1988, p.638).

D'autres travaux s'attachent à évaluer la nature et l'importance de facteurs comme l'homophilie (Verbrugge, 1977, Coenen-Huther, 1989), ou la durée des relations (Ferrand, 1988, 1989). S.N. Eisenstadt \& L. Roniger (1984), en comparant l'amitié avec d'autres formes de liens, s'interrogent sur le rôle social de l'amitié; ils la considèrent comme une "institution sociale non institutionalisée".

Les grandes enquêtes quantitatives qui ont été récemment menées, et qui témoignent d'ailleurs d'un intérêt grandissant pour les relations interpersonnelles $\left({ }^{5}\right)$, fournissent un matériau considérable, centré surtout sur la description du réseau personnel et la recherche des variables qui le déterminent (Héran, 1987, 1988, 1989, 1990). Mais on note qu'elles tiennent peu compte des interrogations et des avancées des études plus qualitatives qui, parfois en-dehors du champ sociologique, essaient de préciser le contenu des relations, leurs rapports avec les pratiques d'échanges, la façon dont elles sont définies par les acteurs. En effet, dans ces enquêtes, la qualité des relations, leur signification pour l'individu, les degrés et les formes d'implication qu'elles mettent en oeuvre, ont été majoritairement soit ignorés, soit déduits de critères dont la pertinence n'est pas démontrée: on range dans la case "amis" des personnes qui se confient entre elles, qui échangent des services ou qui partent en vacances ensemble, ou alors on compte les personnes désignées comme amis par les individus interrogés, sans chercher à approfondir ce que recouvre cette désignation. Les processus d'apposition du label "ami", mis en oeuvre par les individus en interaction, sont soit ignorés, soit "naturalisés" $\left({ }^{6}\right)$.

Cependant, certaines enquêtes ont montré les écarts qui peuvent exister entre réseaux affectifs et réseaux instrumentaux $\left({ }^{7}\right)$, ouvrant ainsi la voie à la recherche approfondie du lien entre fonction et qualité des relations, en particulier telles qu'elles sont perçues et vécues par les acteurs.

Au carrefour entre ces disciplines, entre perspectives et ces points de vue, on peut plaider pour

\footnotetext{
4 On trouve quelques ouvrages qui l'abordent de front, mais qui relèvent plus de l'essai sociologique, philosophique ou journalistique (Alberoni, 1984, Telfer, 1970, Pogrebin, 1987) que de recherches basées sur des enquêtes. 
une problématique qui intègre une pluralité de dimensions, afin de reconstruire l'objet "amitié" dans ses manifestations sociales.

Il s'agit de prendre en compte les milieux concrets (l'entreprise, le quartier, le club de football...) dans lesquels prennent naissance et se jouent les relations, et de définir les caractéristiques contextuelles qui sont pertinentes pour les liens interpersonnels. On chercher aussi à qualifier ces relations, à en construire une typologie sur la base de critères qui, empiriquement, se sont avérés distinctifs $\left(^{8}\right.$ ). On pourra reconstituer le réseau individuel comme un système complexe de relations, et, par la comparaison, rechercher les variables qui en déterminent la structuration. Les processus interactifs, les "histoires des relations", leurs étapes, les seuils importants, leurs évolutions seront aussi étudiés. Enfin, il faudra prendre en considération les représentations sociales de l'amitié, les façons dont les acteurs perçoivent, classent, et mettent en oeuvre leurs relations.

C'est cette dernière dimension, sans doute la moins souvent étudiée à propos de l'amitié précisément, qui sera ici explorée.

S. Moscovici (1976, lè éd. 1961) a entrepris de préciser et de formaliser la notion de représentations sociales. Les anthropologues en sont depuis longtemps familiers, l'étude des systèmes de pensée et de classement "indigènes" étant au coeur de leur problématique. C'est une notion particulièrement multiforme (Doise, 1985), qui articule des niveaux divers, et prend des sens différents selon l'application particulière qu'on lui fait subir $\left(^{9}\right)$. Il s'agit simplement ici de repérer les divers niveaux de référence auxquels font appel les personnes lorsqu'elles parlent de l'amitié, comment s'articulent entre eux ces niveaux, et quelles variables peuvent en marquer les différences.

Lorsqu'on étudie l'amitié, la tâche est à la fois facilitée et compliquée.

Elle est facilitée par la séparation qui de fait isole l'amitié des autres relations dans les représentations des acteurs, et qui permet de la traiter dans une relative autonomie. En effet, il est surprenant de constater à quel point les évocations de l'amitié sont radicalement distinguées de celles des autres relations des personnes. La définition de l'amitié, ses limites, et les personnes avec qui l'individu se déclare ami, semblent être clairement identifiées et stabilisées, alors que pour les autres relations les désignations (copain, connaissance, camarade...) sont beaucoup plus floues et mouvantes, y compris dans le discours d'une même personne. S'il existe un continuum dans l'intensité des relations interindividuelles, l'amitié n'en est pas un pôle, elle se distingue plutôt de l'ensemble des autres relations. Je propose de la traiter de façon autonome, afin d'interroger ensuite son rapport avec les autres types de liens, leurs limites respectives, leurs oppositions. On peut ainsi, pour comprendre ce qu'est une relation d'amitié, étudier les rapports et les différences entre l'ami et le frère, entre l'ami et le copain, le collègue, etc. ${ }^{10}$ ); mais aussi entre des personnes précises, nommées, en posant des questions du type: "quelles sont les différences

\footnotetext{
8 Cf à cet égard la critique qu'opère P. V. Marsden (1984) de la pertinence de la fréquence des interactions comme indicateur de la force du lien.

Pour S. Moscovici (1961), ce sont: "des systèmes de valeurs, des idées, et des pratiques dont la fonction est double: en premier lieu, établir un ordre qui permettra aux individus de s'orienter et de mâtriser leur environnement matériel, ensuite faciliter la communication entre les membres d'une communauté en leur procurant un code pour désigner et classifier les différents aspects de leur monde et de leur histoire individuelle et de groupe".

...voire entre l'ami et l'amant. Cette question de la possibilité de "sexualisation" de la relation d'amitié offre un intérêt certain, et l'on voit des chercheurs y porter aujourd'hui leur attention (Ferrand, 1990). Nous l'éviterons ici, dans la mesure où "a priori", dans le sens commun, le terme "amitié" exclut les relations sexuelles (même si l'utilisation d'expressions comme "mon petit ami", ou "mon copain" pour désigner une relation sexualisée contribue à brouiller les cartes), comme il exclut en français les relations de parenté.
} 
entre votre relation avec $\mathrm{x}$ et celle avec $\mathrm{y}$ ?"... Ainsi peuvent se mettre en place les oppositions et les liens entre des relations dont je fais l'hypothèse (à la suite de G. Allan (1979) et d'A. Ferrand (1985)) qu'elles forment système.

L'étude de l'amitié est par contre compliquée par son apparent statut d'évidence, voire son caractère perçu comme "naturel", tant par la plupart des sociologues que par les acteurs sociaux euxmêmes. C'est d'ailleurs sans doute ce qui a contribué à conduire tant de chercheurs à la considérer comme une catégorie "allant de soi", sans chercher à voir ce qu'elle recouvrait.

On retrouve cette apparente évidence de l'amitié au niveau du sens commun. La question "pourquoi est-on amis?" est surprenante. L'amitié (comme l'amour) est en effet généralement perçue comme inexprimable, relevant de l'irréductible individualité; "par ce que c'estoit luy; par ce que c'estoit moy", disait Montaigne, parlant aussi de "je ne sçay quelle force inexplicable et fatale, médiatrice de cette union". Pour $\mathrm{M}$. Aymard, l'archétype de l'amitié de Montaigne est voisin de l'amour $\left({ }^{11}\right)$. Quoi qu'il en soit, pour être "authentique", l'amitié est perçue comme devant être dégagée des contraintes sociales.

Or, l'amitié est aussi sociale. Pour G. Simmel (1908, trad.1950), la relation interindividuelle est la forme fondamentale de l'interaction sociale, elle-même au coeur de la définition de la société. L'amitié est reconnue socialement, autorisée, normée. On montre qu'elle est soumise à des "règles de convenance" $\left({ }^{12}\right)$, à des déterminants sociaux (Fischer, 1982; Verbrugge, 1977; Schutte \& Light, 1978; Huckfeldt, 1983 ...). Elle répond aussi à des classements sociaux préférentiels (Cf la notion d'homophilie). Bien que dans nos sociétés elle ne soit pas institutionalisée, ritualisée, elle n'en est pas moins une institution (Allan, 1979, Hays 1988), en ce qu'elle est socialement, culturellement objectivée. L'amitié ne concerne pas que les deux amis, leur relation est reconnue par l'ensemble de la société (Mc Call, 1988) $\left({ }^{13}\right)$. Même si, dans la tension qu'elle manifeste entre individu et société, elle se montre plutôt comme "l'inverse de l'ordre" (Ferrand, 1990), elle s'accomode de cet ordre $\left({ }^{14}\right)$. S'insérant dans les interstices de l'ordre établi qui cependant la reconnaît, lui autorise cette place, elle contribue à le stabiliser, à le perpétuer en occupant justement cet espace de marge (donc de danger social potentiel) entre individualité et société. C'est la thèse que proposent Eisenstadt \& Roniger (1984), en mettant en avant son rôle de régulation sociale.

Socialement déterminée, normée, canalisée, l'amitié n'en est pas moins perçue comme fondamentalement a-sociale. Il s'agit maintenant d'explorer dans le détail ces représentations de l'amitié que proposent les acteurs sociaux.

\footnotetext{
11 "Mais si l'amitié est ainsi proposée comme référence ultime dans le champ d'une affectivité qui s'identifie avec l'exercice de notre "liberté volontaire", c'est qu'elle parle d'elle-même le langage de l'amour. Elle nâ̂t d'un coup de foudre, à la première rencontre, "entre deux hommes faits", refusant de "se régler au patron des amitiés molles et régulières, auxquelles il faut tant de précautions de longue et préalable conversation" ". M. Aymard, 1986, p.463.

12 C'est la traduction que propose A. Ferrand (1985) pour les "rules of relevancy" définies par G. Allan (1979).

13 "When persons say they are friends, usually they can point to cultural images, rules of conduct, and customary modes of behavior to confirm their claims. This is likely to be most apparent when one or both parties find their relationship questioned by outsiders. At these times there will be an appeal to standards, rules, or "facts" by which persons can validate whether or not they are, indeed, "friends". (Suttles, 1970, p.98)

14 "C'est un modèle de l'accommodement oppositionnel qui pourrait être développé: l'amitié est a-fonctionnelle du point de vue de l'ordre social établi, elle ne joue aucun "rôle", ne sert à rien. Cependant elle est, elle existe et dure - affirmation désabusée et limitée qu'un autre ordre relationnel est possible." (Ferrand, 1990).
} 
Que se passe-t-il lorsqu'au cours d'une interview les personnes sont invitées à parler de l'amitié? Dans l'enquête que j'ai réalisée auprès d'ouvriers et employés travaillant dans les quartiers Nord de Marseille, à la question "qu'est-ce que c'est pour vous un ami?", la réponse venait sans hésitation. Par contre, lorsqu'il s'agissait ensuite d'expliquer des amitiés particulières avec des personnes nommées ("pourquoi x est-il votre ami?"), la réponse était beaucoup plus difficile à obtenir, et elle s'écartait souvent de la définition de l'ami en général donnée auparavant. Je demandais ensuite de raconter comment s'était passée la rencontre initiale, ses circonstances, le déroulement de la relation, les seuils importants..

Ce sont ces écarts, ces attitudes différentes dans les interviews, qui m'ont incitée à me pencher sur ces trois différents niveaux de discours: la définition de l'ami en général, la description d'amitiés précises, et le récit de la fondation et de l'évolution de la relation.

\section{-1- Représentations de l'amitié, schèmes et variations}

A un premier niveau, l'interviewé répond à une question générale sur un "personnage-type", l'ami. Le fait qu'il soit à même de répondre rapidement nous fait penser que, s'il n'est pas habitué à formuler ce qu'est pour lui un ami, il dispose néanmoins de référents fixés, stabilisés, qu'il peut mobiliser aisément. C'est ce que D. Sperber (1989) appelle "les représentations culturelles". Sans préjuger d'une relation directe d'induction, on remarque par exemple que ces référents culturels sont largement présents dans la littérature. On y voit s'épanouir les amitiés "extraordinaires", bravant le temps et les obstacles sociaux pour faire triompher l'irréductible individualité des héros. L'amitié (mais l'archétype de cette figure est bien sûr l'amour) y est souvent confrontée à l'ordre social. A. Silver (1989) parle d"'idéal de l'amitié", en reconstituant la logique de son contenu tel qu'il apparait dans les sociétés urbaines occidentales actuelles: l'amitié se construirait dans l'inversion de l'ordre social $\left({ }^{15}\right)$, en reposant toute sur l'interpersonnel ("true or 'real' selves").

On pourrait parler ici de figure idéalisée, de "prototype" dans le sens que lui donne G.P. Ginsburg (1988) $\left({ }^{16}\right)$. Ce prototype ne fonctionne pas comme un modèle, il ne détermine pas directement les relations, mais il construit, sous forme de "cas", d'"exemples", des catégories de situations et de personnes, catégories qui peuvent rendre compte des conduites et qui sont socialement reconnues (les psychosociologues insistent sur leur rôle dans la communication). C'est ce mode de référence qui, je pense, apparaît dans les réponses à la question "qu'est-ce que c'est pour vous un ami?".

Ces réponses ne sont pourtant pas univoques, on y trouve des variations. On peut se référer ici au schéma des représentations sociales que propose C. Flament (1989). Sur ce schéma, les représentations d'un objet sont centrées autour d'un "noyau central", qui est le "lieu de cohérence" des représentations, qui en organise les schémes périphériques. Ces schémes périphériques, moins abstraits que ceux qui constituent le noyau central, "assurent le fonctionnement quasi instantané de la représentation comme grille de décryptage d'une situation: ils indiquent, de façon parfois très spécifique, ce qui est normal (et par contraste, ce qui ne l'est pas), et donc, ce qu'il faut faire comprendre, mémoriser... Ces schèmes normaux permettent à la représentation de fonctionner économiquement, sans qu'il soit besoin, à chaque instant, d'analyser la situation par rapport au principe organisateur qu'est le noyau central" (Flament, 1989, p. 209). Ce noyau central est plus stable que la périphérie, il résiste aux changements: "En fait, la périphérie de la représentation sert de zone tampon entre une réalité qui la met en cause, et un noyau central qui ne doit pas changer facilement. Les désaccords de la réalité sont absorbés par les schèmes périphériques, qui, ainsi, assurent la stabilité (relative) de la représentation" (p.210). Il donne l'exemple de la représentation

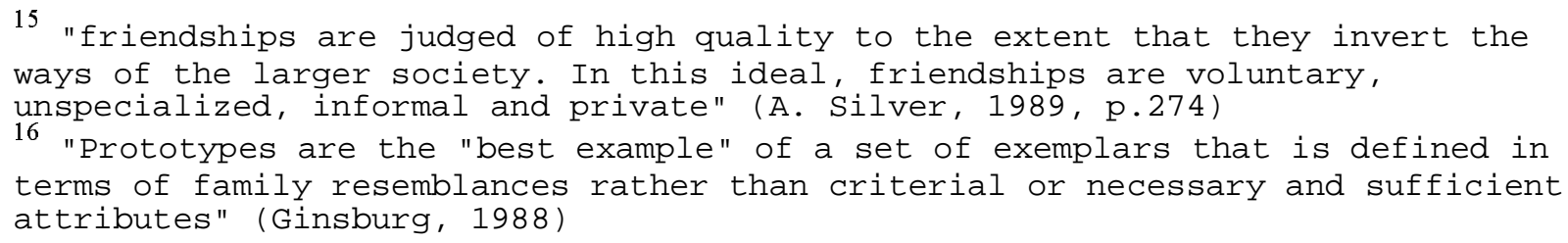


du "groupe idéal", dont le noyau central est constitué par les items "amitié" et "pas de hiérarchie", et montre que litem "une grande convergence d'opinions" est par contre un schème périphérique, dont la mise en cause ne suscite pas le rejet de la grille de décryptage. La zone périphérique autorise aussi une diversité dans l'actualisation des représentations: "Deux sous-populations peuvent avoir, d'un objet donné, une même représentation (c'est-à-dire un même noyau central de la représentation) et, pour des raisons circonstancielles (notamment les pratiques individuelles), des schèmes périphériques inégalement activés d'où des discours différents" (p. 216).

Ce sont ces variations dans la représentation de l'amitié en général que je voudrais mesurer ici, en étudiant les réponses à la question sur la définition de l'ami.

Deux corpus de données recueillis dans des conditions différentes sont analysés:

- d'une part les réponses à la question "qu'est-ce que c'est pour vous un ami?" posée dans l'enquête par entretiens semi-directifs que j'ai réalisés auprès de 58 ouvriers, employés et cadres, hommes et femmes, travaillant dans 5 entreprises $\left({ }^{17}\right)$ situées dans les quartiers Nord de Marseille.

- d'autre part les réponses à la question ouverte "Pour vous, qu'est-ce qu'une amie ou un ami?", posée dans le questionnaire d'une enquête menée par Hugues Lagrange et Sébastian Roché à Grenoble $\left({ }^{18}\right)$, auprès de 1700 Dauphinois habitant en milieu urbain et semi-rural. Je n'ai pu en traiter que 256; ceci contitue bien sûr un sous-échantillon limité, mais il permettra la confrontation avec les tendances que j'ai pu repérer dans ma propre enquête.

Ces deux enquêtes nous fournissent des matériaux différents. Si la question de l'enquête de Grenoble était ouverte (au sein d'un questionnaire fermé par ailleurs), les personnes ne pouvaient guère s'y exprimer par plus d'une ou deux phrases. L'enquête Marseillaise se base sur des interviews relativement longues (deux heures en moyenne), les personnes pouvant largement développer et préciser leurs propositions. J'ai cumulé les définitions recueillies dans ces deux enquêtes pour former 10 catégories, construites empiriquement, et qui permettent la confrontation entre les résultats. Ces catégories ne sont pas systématiques ni exclusives.

Dans la majorité des cas les réponses sont multiples, même dans l'enquête de Grenoble. Les personnes donnent souvent plusieurs définitions successives. J'ai tenu à distinguer la première réponse des suivantes éventuelles. On peut donc repérer précisément les variations dûes à une "poursuite" au-delà de la première proposition spontanément privilégiée.

Je commencerai par décrire ces catégories, préciser leur contenu, et étudier leur distribution selon quelques variables au sein de la population interviewée à Marseille. On pourra ensuite confronter cette distribution avec celle qui ressort de l'enquête de Grenoble, plus susceptible de quantifications.

Je classe ici ces définitions dans l'ordre de leur importance dans l'enquête marseillaise.

- La définition qui s'impose majoritairement à Marseille pourrait être intitulée "le drame". Pour ces interviewés, les amis sont "ceux sur qui l'on peut compter en cas de problème grave"

"Un ami, c'est celui qui sera là quand vous aurez vraiment besoin de quelque chose. Vous pouvez compter sur lui en cas de coup dur"

\footnotetext{
17 Il s'agit d'une entreprise de protection de métaux, d'une semoulerie, d'une entreprise de distribution pharmaceutique, d'une Caisse d'allocations familiales, et d'un supermarché.

18 Lagrange H., Roché S., 1988, Baby alone in Babylone, CERAT, Institut d'études politiques de Grenoble. Je les remercie ici de m'avoir permis de dépouiller ces réponses.
} 
"L'amitié... il n'y a pas beaucoup de gens avec qui on partage notre difficulté et notre besoin. On demande quelque chose... si il m'arrive quelque chose, je le dis, si il le prend au sérieux, ça c'est l'ami"

Les amis sont ceux qui répondent toujours (i.e. sans incertitude) à l'appel. On voit très souvent les amis associés aux mauvais moments, les bons étant partagés avec les "simples copains". C'est aussi la possibilité de sacrifice.

On trouve également l'idée d'un test: c'est là que l'on reconnaît les "vrais" amis, qu'on les distingue des autres.

"Il y a les collègues de travail, et puis l'amitié, je pense que c'est pas pareil. Je pense que les amis, on les voit quand on a des mauvais moments, des problèmes. Peut-être que les collègues de travail aussi, hein, attention, mais enfin je pense qu'il faut qu'il y ait quelque chose qui se concrétise pour vraiment le voir. Je pense que s'il y avait un cas, avec un collègue de travail, il y aurait peut-être ça. Parce que c'est toujours dans le sens... si on a un problème, surtout malheureux; c'est pas quand on est heureux, ces choses là. C'est dans ces moments malheureux qu'on voit vraiment ses véritables amis, et ses collègues. Parce que des fois on est beaucoup entourés et tout, et quand il se passe certaines choses, l'entourage diminue, et c'est là que... ceux qui restent, c'est les véritables amis"

Cette définition est dominante chez les ouvriers, elle apparait majoritairement comme première définition donnée. On la trouve aussi présente chez les employés, plus chez des gens relativement âgés, non diplômés, que chez les jeunes. Des cadres la proposent aussi, mais plutôt en seconde position: elle n'est pas spontanément privilégiée. Ce sont des cadres âgés, non diplômés, qui ont été promus "à l'ancienneté", et sont pour la plupart d'origine ouvrière. De façon générale, les hommes la proposent plus que les femmes.

La pregnance de cette définition, mais surtout la façon dont les personnes l'érigent en "test", peut nous faire penser qu'elle fait partie du "noyau central" de la représentation de l'amitié. Elle structure la cohésion de la catégorie, en ce qu'elle en constitue un critère d'appartenance. "La théorie structurale du noyau central permet de prévoir que la mise en cause d'un élément du noyau entraine le rejet de la représentation "groupe idéal" (ici, "ami") comme grille de décryptage de la situation (un groupe égalitaire ne peut être hiérarchisé" (ici, un ami ne peut pas faillir en cas de coup dur)) (Flament, 1989, p.208). D'autres définitions, moins décisives, et non exclusives, peuvent être apparentées à des schèmes périphériques.

- Une autre catégorie est celle qui définit l'amitié comme une relation de confidence.

"Une amie, c'est une personne à qui je confie... avec qui j'ai des relations plus que des sorties, je peux lui confier certaines choses sur ma vie personnelle; à qui je peux tout raconter, qui m'écoutera, qui me donnera des conseils, sa façon de voir les choses. Je n'ai pas d'amis dans le travail car j'ai peur des racontars, que ça soit répété".

On trouve là l'idée de sceau du secret. La confidence implique souvent aussi la tolérance, le respect, l'acceptation des différences; "on n'est pas jugé".

Ce sont les employés qui proposent surtout cette définition, surtout les femmes, d'âge moyen, et peu diplômées.

Contrairement à la définition en terme de "drame", elle n'est pas exclusive: des personnes, des hommes principalement, ont des amis avec qui ils n'échangent pas de confidences. Les catégories qui suivent ne semblent pas, de la même façon, faire partie du noyau central. 
- La comparaison entre l'amitié et la relation familiale est souvent proposée

"Un ami, c'est comme un frère que j'aurais choisi. Je peux lui faire confiance, comme à mon frère ou à mes parents"

Parfois, la comparaison se fait au détriment de la famille

"Un ami, c'est quelqu'un sur qui on peut compter. Peut-être plus que sur la famille. On a besoin de quelque chose, on se tourne vers eux. Que la famille, des fois on se tourne vers eux, eh bien... c'est non. C'est sur les amis qu'on peut compter. Des fois on dit: "les frères et les soeurs... mais moi j'ai mon amie, c'est sûr, ça fait 22 ans qu'on se connaît, c'est elle ma soeur"

Ce rapprochement est opéré par les ouvriers, non diplômés, autant masculins que féminins.

- On trouve aussi une catégorie rendant compte de la "facilité" de la relation: "Il n'y a pas de gêne", "on se sent à l'aise", "on n'a pas besoin de faire des efforts", "on passe sans être invité"; "on n'a pas besoin de rendre des comptes"; "On n'a pas de devoirs".

"Quand on est amis, on ne s'invite pas, les autres viennent, on mange ce qu'il y a. On peut passer à onze heures du soir. C'est ça qui fait les amis. Mais si ça dérange, on le dit, et il n'y a pas de problèmes".

Ce sont là aussi surtout les cadres diplômés qui proposent cette définition. Elle semble aussi être réservée aux jeunes.

- La définition reposant sur la notion de "confiance" nous a été relativement peu donnée telle quelle dans cette enquête. Elle implique, pour les personnes, (nous nous appuyons aussi sur les réponses recueillies dans l'enquête de Grenoble), la franchise, la sincérité, l'honnêteté, le désintéressement, l'absence de jalousie.

"Un vrai ami, c'est la confiance. Moi je n'ai confiance en personne, sauf mon père et ma mère. Les vrais amis, c'est quand même moins que les parents, pour la confiance. Et l'amitié, c'est la confiance".

On peut penser cependant que cette notion de confiance est impliquée dans de nombreux autres items, tels la confidence, le drame... Nous y reviendrons.

- La "proximité interindividuelle" marque une ressemblance qui est posée sur un plan personnel; "on a le même caractère, les mêmes goûts, les mêmes idées, des affinités personnelles, on se comprend, on a de la complicité".

"Avec un ami, il n'y a pas besoin de parler pour se comprendre. On a des idées communes, une complicité. Si je vois qu'il fait une remarque que j'aurais faite, ça fait "tilt". En fait, il faut qu'il me ressemble"

La proximité interindividuelle est mise en avant surtout par des jeunes, plutôt diplômés, employés masculins pour la plupart.

- Une autre catégorie de définitions est basée sur les échanges affectifs: "on est bien ensemble, on a du plaisir à se voir, on s'aime".

"Les amis c'est ceux avec qui on est bien, qu'on est toujours contents de voir, avec qui on a plaisir 
à faire quantité de choses différentes

Ce sont presque exclusivement les cadres qui proposent cette conception de l'ami. On trouve aussi quelques employées, diplômées également. C'est d'ailleurs une définition plus féminine que masculine. Les femmes cadres la donnent plus que leurs collègues masculins, qui la mettent parfois en seconde position.

- Certains privilégient la permanence, la présence: l'ami est celui qui est là, toujours disponible, toujours prêt à vous recevoir. Les jeunes y associent principalement le partage d'activités, alors que pour les gens plus âgés, c'est surtout une compagnie, un antidote à la solitude.

"Un ami, tu l'as 24 heures sur 24. L'autre, tu l'as dans le besoin, c'est vrai, il vient quand tu as un coup dur, mais il me semble que l'ami à part entière, c'est celui que tu as 24 heures sur 24; celui qui est là en permanence pour t'épauler."

Cette définition est relativement peu proposée. Les femmes semblent la privilégier plus que les hommes.

- La "proximité sociale" peut aussi définir l'amitié: il s'agit là d'une ressemblance sur le plan social et culturel. Ce sont des "gens comme nous", "on est au même niveau", "on a la même éducation", "on est du même milieu". Elle nous a été très peu proposée à Marseille, du moins au niveau d'une définition générale de l'amitié.

- La définition qui privilégie le "long terme", l'inscription de la relation dans le temps a été très peu mise en avant dans notre enquête. Là, l'ami "c'est celui qui me connaît bien", et cela demande du temps, "on se connaît depuis longtemps".

"C'est difficile, les amis. Il y a des amis qu'on voit rarement mais ce sont des amis. C'est le passé qui fait l'amitié, ce qu'on a vécu en commun"

Grenobloise.

On peut maintenant comparer la distribution de ces définitions avec celle qui ressort de l'enquête

Nous avons dressé le tableau des pourcentages obtenus par chaque définition ( $\mathrm{n}=256)$

Le pourcentage global est calculé par concaténation de la première définition et des deux suivantes éventuelles. Pour les autres, on calcule le pourcentage de chaque définition parmi les définitions données en premier rang d'apparition dans la réponse, puis en second, en troisième rang. Les pourcentages des définitions données en 2 è et 3è position sont calculés sur les choix exprimés.

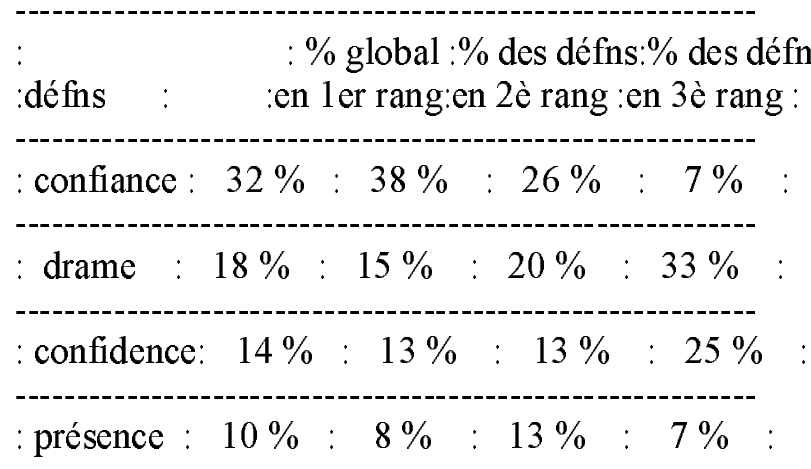




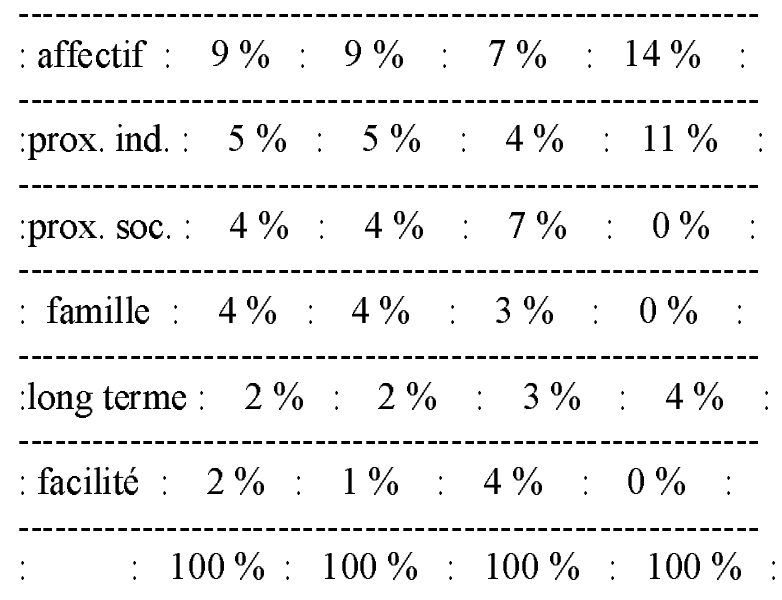

Dans un premier temps, on remarque que dans l'enquête de Grenoble la définition basée sur la confiance est très majoritairement privilégiée.

Il est intéressant aussi de remarquer que certaines définitions (le "drame", la "confidence", la "proximité interindividuelle", "l'affectif") sont plutôt proposées en 3è position. Ce sont donc des définitions de l'amitié qui ne sont pas données d'emblée, mais dans un second temps, comme en complément; alors que la définition basée sur la confiance est surtout proposée d'emblée.

En ce qui concerne la distribution selon les variables de sexe, d'âge, de profession, de niveau de diplôme, on retrouve globalement les mêmes tendances que dans l'enquête marseillaise.

La définition impliquant la confiance est également répartie selon les variables: on ne trouve aucun écart remarquable par rapport au pourcentage global. Il semblerait donc que ce soit une définition relativement consensuelle, régulièrement distribuée socialement.

On retrouve par ailleurs la division entre les ouvriers, qui privilégient le "drame" et la comparaison avec le lien familial, et délaissent relativement (i.e le pourcentage est nettement inférieur au pourcentage global) l'affectif, la proximité interindividuelle et la confidence; les employés qui mettent surtout en avant la confidence, puis le long terme; et les cadres qui proposent très nettement plus l'affectif, et se réfêrent également à la proximité sociale, délaissent le drame.

La division sexuelle est nette: les hommes privilégient relativement plus la définition "drame" et la comparaison avec la famille; les femmes, la confiance et l'affectif.

En ce qui concerne l'âge, le drame intervient plus après 45 ans, ainsi que l'affectif; les jeunes privilégient plus la proximité interindividuelle, la présence (activités partagées), la facilité. Les 35-44 ans sont plus attachés à la confidence, la proximité sociale (plus marquante à cet âge?).

Si l'on croise le sexe avec la profession, on remarque que les femmes cadres choisissent plus l'affectif et le long terme que leurs collègues masculins, et moins la proximité sociale. Les femmes employées ne se distinguent pas nettement des hommes. Les ouvrières choisissent relativement plus l'affectif que les ouvriers, bien que tous proposent d'abord le drame.

$\mathrm{Au}$ niveau du bagage scolaire, les personnes sans diplôme ainsi que les titulaires de diplômes professionnels privilégient le drame et la famille, et négligent la proximité sociale et l'affectif. également. Ceux qui ont le BEPC et BEPS privilégient la proximité interindividuelle, la confiance et le long terme. Les bacheliers insistent sur la proximité sociale, et la proximité interindividuelle, négligent le drame et la confidence. Les licenciés privilégient nettement l'affectif, délaissent le drame et la famille.

On retrouve assez bien les tendances liées à la profession. Si l'on croise la profession avec le diplôme, on remarque que les cadres choisissent parfois le drame lorsqu'ils sont peu diplômés (au-dessous du bac: cadres par promotion interne). Ce n'est en fait qu'au-dessus de la licence qu'ils proposent massivement l'affectif. Le niveau de diplôme intervient moins pour les employés, et les ouvriers possèdent trop rarement un diplôme pour que le compte soit significatif. 
On peut penser, très schématiquement, que les ouvriers sont plus attentifs à la sécurité (réponse assurée en cas d'appel, de drame; rapprochement d'un rôle "assigné", celui du frère); que les employés privilégient le versant de la proximité interpersonnelle, ce qui est du côté de la compréhension mutuelle, de la ressemblance, de l'intimité. Les cadres eux font intervenir "l'affectif", tout en restant attentifs à la proximité sociale.

La moitié des personnes ayant donné plus d'une définition, il est intéressant d'étudier leurs cooccurrences, du moins pour celles qui obtiennent un score significatif:

Les personnes qui donnent la définition de "la confiance", l'associent avec toutes les autres de façon assez répartie, sans qu'il apparaisse de nette priorité.

On remarque par ailleurs trois couples fortement liés: facilité-drame, présence-confidence et affectifproximité interindividuelle. La confidence est donc liée à la quotidienneté, s'éloignant ainsi du drame qui relève de l'exceptionnel mais implique une absence de règles formelles de relations, de devoirs. L'échange affectif serait associé à la proximité interindividuelle (on s'aime parce qu'on se ressemble).

La définition basée sur la notion de confiance, est, on l'a vu, la plus largement proposée à Grenoble. Mais elle est aussi la mieux répartie, la moins variable. Toutes les catégories de personnes la proposent spontanément, dont la plupart en première position et la moitié comme seule définition. Le fait qu'on la retrouve moins dans l'enquête de Marseille peut sans doute être rapproché des différences dans les modes de recueil des données: entre un questionnaire fermé (à Grenoble) que l'on remplit assez rapidement, dans un mode de discours globalement succinct, et une interview longue et approfondie (à Marseille), qui demande un investissement plus lourd, et provoque des réponses plus spécifiques, plus précises... $\left({ }^{19}\right)$.

Il me semble en effet que l'on peut considérer la définition basée sur la confiance comme une définition à la fois plus large et plus centrale. Elle englobe en quelque sorte les autres définitions, au niveau du sens. Elle en donne un résumé qui concentre le noyau positif, culturellement valorisé, de l'amitié. Si l'on reprend le schéma de $\mathrm{C}$. Flament, la confiance serait également au coeur du "noyau central" de la définition. On peut la comparer avec la notion de "drame" qui est également dans ce noyau. Alors que la définition qui repose sur ce "test" de l'attitude de l'ami en cas de coup dur peut être assimilée à un "prototype", un "exemple", un "cas" (Ginsburg, 1988, Semin, 1989), la notion de confiance serait le terme englobant, général, qui résume et concentre la condition de la relation d'amitié plutôt que d'en donner une illustration prototypique.

Les autres définitions relèveraient des "schèmes périphériques" (Flament, 1989), du "halo flou" (Boltanski, 1982), des "contours flous" des représentations culturelles (Sperber, 1989) $\left({ }^{20}\right)$. Ces définitions ont à voir avec la confiance mais en donnent des éclairages précis et divers. Ce sont elles qui varient, qui sont distribuées différemment entre femmes et hommes, entre jeunes et vieux, entre cadres et ouvriers $\left.{ }^{21}\right)$.

19

19 Si l'on peut s'interroger sur l'éventualité de variations régionales, nous n'avons pas les moyens de les mesurer ici, les différences dans le mode de recueil des données nous paraissant plus déterminantes. D'autres écarts dans l'ordre majoritaire des définitions recueillies peuvent relever de différences entre les échantillons de Marseille et de Grenoble. On se souviendra qu'il ne s'agit ici que de repérer des grandes tendances.

20 On remarquera la convergence des diverses disciplines...

21

C. Flament remarque, à propos d'une étude sur les représentations de la culture: "L'étude suggère que toute la population a une unique représentation de la culture (en termes de noyau central), mais que les divers individus ont, pour des raisons en partie sociales, en partie personnelles, des pratiques culturelles plus ou moins intenses, d'où des niveaux variés d'activation de certains schèmes périphériques et des discours différents sur la culture." (1989, p.216) 
Nous avons fait l'hypothèse plus haut de l'appartenance de la définition en terme de "drame" au "noyau central" de la représentation de l'amitié. Or, on constate des variations dans la référence à cette définition: les ouvriers la proposent plus que les cadres. Contrairement aux enquêtes mises en oeuvre par les psychosociologues, la nôtre ne propose pas de définitions, la question (à Marseille comme à Grenoble) est ouverte. Il ne s'agit donc pas de marquer un accord mais de privilégier spontanément une définition. Il reste que si l'on reprenait les dix catégories isolées ici, sous forme de questions fermées, l'accord avec l'item "l'ami est celui qui ne fait jamais défaut en cas de coup dur", serait, c'est mon hypothèse, assez général. Les variations ici dégagées relèvent plutôt, pour cette définition, d'une plus ou moins grande distanciation avec cet élément du noyau central, d'une propension différente à l'exprimer spontanément tel quel. $\left({ }^{22}\right)$

L'étude du lien étroit qui associe l'amitié avec la confiance est développée par S.N. Eisenstadt \& L. Roniger (1984) et A. Silver (1989). L'interpersonnalité, l'intimité de la relation d'amitié suppose une connaissance de l'autre sur laquelle repose la confiance, confiance qui cependant ne se réduit pas à cette connaissance. Pour G. Simmel, la confiance est intermédiaire entre connaissance et ignorance à propos d'une personne (Simmel, 1950, p.318), pour A. Silver la confiance dépasse l'expérience $\left(^{23}\right.$ ). Elle n'a en tout cas pas toujours besoin de temps pour s'établir; les entretiens nous fournissent nombre d'exemples d"'amitiés-coup de foudre", où dominent l'"instinct", "le flash", le "tilt"... La confiance, pour A. Silver, permet une réduction de l'incertitude à propos de l'autre.

Cette réduction de l'incertitude est ce à quoi semblent s'attacher plus particulièrement les ouvriers, qui insistent sur la dimension sécurisante de l'amitié: en situation exceptionnelle, en cas de drame, l'ami est celui qui répond toujours en cas d'appel, ou même sans qu'il y ait appel.

"Les amis, il n'y a pas besoin d'y faire appel, ils sont là, on peut compter sur eux. Il n'y a pas besoin de parler pour se comprendre. C'est très important, l'amitié. J'en ai besoin, ça me rassure. J'ai besoin de savoir qu'il y a des gens autour; besoin qu'ils me confortent dans mes décisions."

Le "toujours" renvoie non pas à une dimension temporelle mais à la certitude de la réponse adéquate. Même s'ils se défendent, dans les interviews, d'associer un quelconque intérêt avec la relation d'amitié, en stigmatisant fortement cette attente, il y apparait néanmoins nettement la notion d'un devoir. Devoir qui, s'il n'est pas rempli, s'il y a "déception", "trahison de la confiance", peut être cause de rupture.

Les employés, qui privilégient la dimension de la confidence, s'attachent par là à maximiser directement la connaissance de leur partenaire. La confidence est aussi une insistance sur le caractère privé de la relation: elle est réservée. Elle implique aussi, et les personnes interviewées insistent fortement sur cet aspect, le respect du devoir de secret. Là aussi, la trahison est rédhibitoire. La confidence fait aussi appel à la notion de franchise; on y dit ce qu'on ne dit pas ailleurs, qui n'est habituellement pas dicible, et qui peut faire appel à une compréhension, à une tolérance particulières, même si l'on demande aussi de conforter des opinions ou des attitudes, de réconforter. C'est l'intimité sans fard. S'il y entre une dimension de sécurisation, elle est plus quotidienne, plus privée que pour les ouvriers.

\footnotetext{
22

22 On peut aussi se demander si les ouvriers ne marquent pas, plus que d'autres catégories sociales, une tendance à s'exprimer de façon générale sous forme de "prototypes", d'exemples, de récits. Cette hypothèse n'est pas nouvelle (cf. B. Bernstein, 1975). Ses extensions parfois caricaturales ont été critiquées par W. Labov (1976). Voir aussi la présentation des travaux de Schatzmann et Strauss dans Bourdieu, Chamboredon \& Passeron (1968). Nous n'avons pas les moyens de tester ici cette hypothèse.

23

"The act of trust extends commitment beyond extrapolated experience, by resolving uncertainties about others in the direction of unconditional confidence in their essential qualities and enduring dispositions" (Silver, 1989, p.277)
} 
Les cadres diplômés semblent, eux, mettre en avant la tonalité affective. Il n'y a plus de conditions, plus de demande de sécurisation, plus de devoirs. La confiance se construit dans le sentiment, le plaisir du contact. La proximité sociale, qu'ils mettent également en avant, permet peut-être de faire l'économie d'exigences plus formelles.

En ne considérant que ces trois catégories sociales, qui semblent marquer des tendances nettes dans leurs propositions de définitions de l'amitié (on pourrait aussi comparer les sexes, les âges...), on voit se dessiner au fur et à mesure que l'on "monte" dans les classes sociales, une tendance à privilégier les caractères informel, libre (sans devoirs), privé, proche, intime, de l'amitié.

Ces trois définitions, le drame, la confidence et l'affectif, font en tout cas ressortir le caractère exceptionnel (situations d'exception pour les ouvriers, relations d'exception pour les employés et les cadres), et privé, à l'écart des institutions sociales, de l'amitié.

\section{-2- Descriptions des relations vécues}

On peut maintenant explorer le second niveau de discours, celui qui est recueilli au moment où, dans l'entretien à Marseille, les personnes nous décrivent et comparent entre elles des relations vécues, particularisées, avec des personnes nommées. La réponse à la question "pourquoi x est-il votre ami?" provoque surtout de l'embarras. Selon G. Allan, il n'y aurait pas de mythe expliquant les relations d'amitié $\left({ }^{24}\right)$. Il signale ainsi que les raisons pour lesquelles se construit une amitié n'existent pas dans le discours. Il n'y a pas de niveau de discours permettant de rendre compte d'une relation existante, en train d'exister. On peut raconter sa fondation, expliquer les raisons de sa rupture, mais pas celles de son existence courante. On peut néanmoins recueillir les descriptions de leurs relations que font les personnes au cours de l'interview, en particulier en leur demandant de comparer les liens avec les partenaires réels qu'elles ont qualifiés d'amis ("Est-ce la même amitié avec X et Y? A quoi sont dûes les différences?"). Nous avons donc eu recours à la comparaison pour faciliter l'émergence des traits pertinents.

Il peut paraître surprenant, au premier abord, de les voir alors se détacher de la définition générale qu'ils ont donnée de l'amitié, comme s'ils l'avaient oubliée $\left({ }^{25}\right)$.

Les ouvriers, on l'a vu, définissaient majoritairement l'amitié par le soutien en cas de problème grave. Ils introduisent par ailleurs, dans les descriptions de leurs relations amicales réelles, des schèmes plus liés à l'interindividualité, aux affinités, à l'affectivité, qui se rapprocheraient donc plus des définitions en termes de proximité interindividuelle et de lien affectif. Pour les ouvrières, on voit apparaître largement la confidence.

\footnotetext{
24

24 "Interestingly there are virtually no myths prevalent in our culture to

"explain" friendship attractions" (Allan G., 1979, p. 42)

25 Ce type de divergence n'est pas exceptionnel: "Procédant à l'analyse

segmentaire de ce corpus, on met toujours en évidence des segments de

significations locales très diverses, divergentes, souvent même

contradictoires. On peut considérer deux segments praxiques, ou deux segements

discursifs, ou un segement praxique et un segment discursif; ainsi (Jodelet,

1985), on peut dire que la folie n'est pas contagieuse, et avoir, avec les

fous, des pratiques d'hygiène destinées à prévenir la contagion". (Flament,

1989, p.205). Si nous n'avons pas ici observé directement les pratiques, mais recueili seulement du discours sur les pratiques, l'écart entre la définition de l'amitié en général et la description de l'amitié pratiquée nous paraît suffisant pour y étendre cette analyse.
} 
Les employés privilégiaient dans leurs définitions générales la confidence, la proximité interindividuelle. La différence est moins marquée que pour les ouvriers: ils décrivent également leurs relations réelles comme relevant de ces deux schèmes, y ajoutant le long terme, la présence et "l'affectif". Les employées femmes en particulier centrent très nettement leurs relations réelles sur la confidence et la proximité interindividuelle, mais elles les décrivent plus que les hommes en termes affectifs. Les employés masculins, s'ils proposaient moins que les femmes la confidence comme définition, l'abordent plus dans leurs descriptions de relations réelles; mais il faut noter qu'ils sont, dans ce cas, plus diplômés que leurs homologues féminines.

Les cadres mettaient l'accent d'emblée dans leurs définitions de l'amitié sur l'affectif, la proximité sociale et le long terme, et proposaient parfois "l'aide en cas de coup dur" (pour les cadres non diplômés principalement). Dans la description de leurs relations vécues, ils ajoutent la proximité interindividuelle mais aussi la confidence, pour laquelle les hommes rejoignent presque les femmes.

De façon générale, l'affectif, la proximité interindividuelle, la confidence et la présence apparaissent bien plus dans les descriptions de relations avec des personnes précises que dans la définition de l'amitié "in abstracto", plus extérieure au vécu des individus. Une personne nous a explicitement désigné cette différence:

"Un ami, c'est quelqu'un sur qui on peut compter. Enfin, en apparence. Sur qui on peut compter, tant qu'on a pas d'ennuis, justement, on peut peut-être y compter, mais quand on a des ennuis, c'est à savoir si ce sera vraiment un ami.

Q: C'est un peu un test, cette question d'ennuis?

R: Un test, je sais pas. Ca peut donner une opinion.

Q: parce que vous disiez: quand on a un pépin, c'est là qu'on voit si c'est des amis.

R: Non, ça c'est des idées reçues que l'on a (rire), c'est des idées reçues, ça. Je sais pas, moi j'ai pas eu à vivre cette situation"

Il décrira ensuite ses amitiés comme relevant essentiellement d'une proximité de mentalité, de valeurs.

Il prend donc ses distances par rapport à ce discours "culturellement normé" qu'il a reproduit $\left({ }^{26}\right)$.

D'autre part, comme la quasi-totalité des personnes qui ont proposé la définition de "l'aide en cas de coup dur", il dit ne pas avoir eu à affronter cette expérience. Cette notion de "test" (c'est dans l'adversité que l'on voit qui sont les vrais amis, les "faux" nous abandonnent ou nous déçoivent) semble donc détachée de la réalité vécue, ne pas relever d'un "passage à l'acte", mais plutôt d'une épreuve imaginaire, dont le résultat hypothétique fonctionne bien comme critère décisif pour l'imposition du label "ami".

\section{-3- $\underline{\text { Histoires vécues de l'amitié }}$}

Voyons maintenant ce qu'il en est au niveau de l'expérience racontée des histoires des relations, des circonstances de leur fondation, des seuils importants qu'elles ont traversé. On s'aperçoit alors que d'une façon extrêmement majoritaire, les amis se sont connus dans un moment où l'un des deux traversait un moment difficile (décès d'un parent, problèmes avec les enfants, difficultés conjugales ou affectives, divorce... principalement). Lorsqu'ils se fréquentaient déjà, ce seuil a marqué le passage à une relation d'amitié

\footnotetext{
26 "Même lorsqu'un discours intégré existe, un enseignement de doctrine religieuse par exemple, on aurait tort d'y voir automatiquement l'expression exacte des représentations mentales de ceux qui le tiennent ou l'écoutent: ce discours est lui-même un comportement, souvent un comportement de répétition autant que de communication, un comportement qui doit être compris à partir des croyances et des intentions qui le sous-tendent; il n'est pas à lui-même sa propre explication." (Sperber, 1989, p.123)
} 
"Q: C'est devenu une amie comment, X?

R: Elle a eu de gros problèmes de famille, elle est mariée à un psychiatre, voilà. Je pense que je vous ai donné toute l'explication. Et elle s'est retrouvée une main devant une main derrière, le gars l'avait épousée pour son fric, et une fois qu'il a eu terminé ses études il l'a plantée là, mais assez méchamment, il lui a donné tellement de complexes, il l'a tellement rabaissée que c'était devenu une vraie loque. Et c'est là que j'ai réagi, que je l'ai... disons que l'amitié est devenue plus profonde parce qu'on a été obligées de discuter de certaines choses qui font que après ça devient plus solide, quoi."

On peut se demander pourquoi nombre de personnes, qui donnent une définition de l'amitié centrée sur ces situations dramatiques, et qui ont effectivement fondé des relations amicales sur de telles situations, d'une part ne les mentionnent pas dans la description de leurs amitiés précises, et d'autre part disent n'avoir pas eu l'occasion de vivre la situation de "test" (le drame qui sépare les "vrais amis" des autres). Il peut y avoir plusieurs types de raisons. D'abord, le "drame" est advenu alors qu'ils n'étaient pas encore amis, n'a donc pas été pertinent à l'époque de l'initiation de la relation, et ce n'est que par nos questions qu'ils peuvent en reconstituer la cohérence.

"Les vrais amis, c'est quand on peut faire n'importe quoi, il peut vous arriver n'importe quoi, sans que... on se sent épaulé. Pas forcément absout, mais on peut s'appuyer. Ils peuvent être contre, même, franchement contre.

Q: c'est arrivé qu'effectivement vous ayiez besoin d'eux ou qu'ils aient besoin de vous, et que...

R: Non, non. C'est une hypothèse. Parce que, remarquez, on s'est épaulés, oui, par exemple, celle de la Drôme a eu une fracture du bassin, elle est seule, elle est isolée, elle est venue trois semaines à la maison, sans problème. Voilà, c'est ça que j'appelle des vrais amis."

Ils ne peuvent pas, auparavant, lier ce drame à leur ami actuel, qui leur apporte maintenant essentiellement de la complicité et de l'affection quotidiennes. Ensuite, ce fameux test de "l'ami qui reste quand les malheurs ont éloigné les autres" semble être plus incantatoire que véritablement appliqué en tant que test (la situation dramatique initiale n'est pas reconnue comme test). Il n'est pas pour autant illusoire: son efficacité certaine au niveau des représentations n'a sans doute pas besoin d'être confirmée par l'expérience; on peut très bien "trier" ses amis en leur attribuant une confiance réelle à partir d'un critère "fictif", en tant que l'on dispose de suffisamment d'informations (et c'est ce seuil d'informations qu'il serait intéressant de pouvoir préciser) pour être sûr qu'il réagirait bien en cas de drame.

Beaucoup de personnes, en particulier des cadres, qui n'avaient pas proposé ce schème dans leur définition générale de l'amitié, ont aussi fondé, leur récit en témoigne, leurs relations dans des moments difficiles. Ces expériences initiales ne sont donc pas forcément rapportées au niveau de la représentation globale.

On trouve aussi des cas où la relation a débuté dans des circonstances pas vraiment dramatiques, mais en tout cas exceptionnelles. L'accouchement en est un exemple. J'ai pu voir plusieurs hommes particulièrement sensibles au fait qu'une personne (un homme en particulier) aille visiter leur femme à la clinique, et qui ont situé là le seuil de leur amitié, ou du moins ont attribué à cet évènement la valeur d'un signe, d'une preuve. D'autres situations, plus collectives, sont aussi des ferments d'amitiés. Le stage de formation assuré par l'entreprise, où les employés se retrouvent ensemble et isolés du reste du monde, est caractéristique de ce type de moment privilégié.

"Vous savez, pendant ces formations, on vit intensément, on doit dormir trois ou quatre heures par nuit, on passe une vingtaine d'heures par jour ensemble. C'est des gens que je voyais dans leurs comportements professionnels et en même temps dans leurs comportements privés, des gens que je m'imaginais, parce que j'étais quand même très jeune à l'époque, que je m'imaginais sans 
problèmes et qui en avaient, et qui à un moment donné me l'exprimaient. Ca m'avait rassuré... (...) On se retrouvait tous célibataires, à ces moments là, tous pareils."

Le service militaire est aussi, c'est bien connu, un moment privilégié de relations, et de relations qui durent parfois bien au-delà du temps de l'armée.

"C'est la relation qui résiste le plus. Je crois que tous les hommes qui ont fait l'armée vous expliqueront pourquoi. Je crois que c'est la période, à l'armée tout le monde est pareil, il n'y a plus de différences. C'est comme le sport, il n'y a plus de riches, plus de pauvres, plus d'intelligents ni de... c'est pareil pour tout le monde, tout le monde est confronté à la même imbécilité ambiante, et ça ne peut que resserrer les liens."

La situation est socialement exceptionnelle, et tend à gommer les repères sociaux. Elle permet à une relation élective, détachée des contingences et des nécessités, des intérêts socialement investis, comme prétend l'être idéalement l'amitié, de trouver un terrain particulièrement favorable, déjà dépouillé.

Les amitiés d'enfance, qui persistent parfois à l'âge adulte bien que le mariage, les enfants et les déménagements en éliminent une bonne part (surtout pour les femmes), sont considérées par les acteurs comme des relations exceptionnelles, dont la principale qualité réside justement dans leur capacité à survivre aux changements de sphères sociales.

"Quand je dis que c'est mon meilleur ami, ça veut dire que c'est celui finalement avec lequel j'aurais eu le plus de facilité pour le perdre de vue."

La "valeur", le principe même de la relation, repose sur cette résistance aux bouleversements, plus que dans la longévité elle-même. On remarque par ailleurs combien la "multiplexité" des relations peut en ellemême être facteur de déclenchement ou de renforcement de leur intensité. C'est parce qu'ils habitent aussi dans le même quartier que des collègues de travail feront plus amplement connaissance.

La majorité des relations d'amitié prend ainsi racine dans des situations "exceptionnelles", hors des cadres et des réponses socialement institués qui balisent la vie ordinaire. Cela ne veut pas dire pour autant que l'amitié soit proprement a-sociale. On sait qu'elle est, comme les autres formes de sociabilité, socialement marquée. Mais les circonstances qui la fondent l'écartent de fait des situations communes. Et ce moment fondateur peut en constituer l'essentiel, qu'il soit traduit ou non dans l'expression d'un "prototype" de la définition de l'amitié. Ce prototype, qui est un élément du noyau central de l'amitié, n'est pas en correspondance immédiate avec les représentations des relations vécues, mais on peut parfois en retrouver le pouvoir structurant dans les récits de fondation des relations.

Le schème central, la figure idéale de l'amitié se concentre par ailleurs dans la notion de confiance. L'expérience de situations exceptionnelles est souvent à l'origine (bien que ce lien soit généralement méconnu par les acteurs) de la confiance. Le caractère privé, interindividuel, affectif, qui constitue la représentation dominante des relations vécues, peut dériver de cette fondation: c'est le drame qui isole l'individu en tant que tel, qui le distingue. L'ami, c'est la personne exceptionnelle dans le moment exceptionnel.

\section{Références bibliographiques:}


- ADAMS B.N., Kinship in an urban setting, Chicago, Markham, 1968

- AGULHON M., Le cercle dans la France bourgeoise, 1810-1848, Etude d'une mutation de sociabilité, Cahiers des Annales, Paris, A. Colin, 1977

- ALBERONI F., L'amitié, Paris, Ramsay, 1984

- ALLAN G.A., A sociology of friendship and kinship, London, Boston, Sydney, G. Allen \& Unwin, 156 p.

- ARGYLE M., The skills, rules, and goals of relationships, in R. Gilmour \& S.W.Duck (eds), The emerging field of personal relationships, 1986, Hillsdale, N.J., Erlbaum, p.23-39

- ARIES P. \& DUBY G. (eds), Histoire de la vie privée, de la première guerre mondiale à nos jours," Paris, Seuil, 1987

- AYMARD M., Amitié et convivialité, in ARIES P. et DUBY G. (eds), Histoire de la vie privée, CHARTIER R. (ed), De la renaissance aux lumières, Tome 3, Paris, Seuil, 1986, p. 455-499.

- BABCHUK N., Primary friends and kin: a study of the associations of middle-class couples, Social Forces, $1965, \mathrm{n}^{\circ} 43, \mathrm{p} .483-493$

- BABCHUK N. \& BATES A., The primary relations of middle-class couples: the study of male dominance, American sociological review, 1963, n²8, p.377-384

- BECKER H.S., Outsiders, Paris, A.M. Métailié, 1985

- BELL C.R., Mobility and the middle-class extended family, Sociology, 1968, vol.2, p.173-184

- BERNARD H.R., SHELLEY G.A., KILLWORTH P., How much of a network does the GSS and RSW dredge up?, Social Networks, $1987, \mathrm{n}^{\circ} 9$, p.49-61.

- BERNSTEIN B., Langage et classes sociales, Paris, Ed. de Minuit, 1975

- BIDART C.,(a) Sociabilités: quelques variables, Revue Française de Sociologie, 1988, XXIX, p.621648.

- BIDART C. (b), Les amis, les semblables et les autres..., Sociabilité et amitié, Colloque de l'AISLF, Genève, 1988

- BOLTANSKI L., Les cadres; la formation d'un groupe social, Paris, Ed. de Minuit, 1982, 523 p.

- BOTT E., Family and social network, London, Tavistock, 1957

- BOURDIEU P., CHAMBOREDON J.C, PASSERON J.C., Le métier de sociologue, Paris, Ed. de Minuit, 1968

- BURT R., Toward a structural theory of action, New York, Academic Press, 1982

- BURT R, A note on strangers, friends and happiness, $\underline{\text { Social networks, }}$ 1987, nº 9 , p.311-331 
- CLARK G. \& S., Friendship in the Christian tradition, in R. Porter \& S. Tomaselli (eds), The dialectics of friendship, London, Routledge, 1989

- COENEN-HUTHER J., Relations d'amitié, mobilité spatiale et mobilité sociale, Espaces et sociétés, $1989, \mathrm{n}^{\circ} 54-55$, p. $50-65$

- COHEN Y.A., Patterns of friendship, in Cohen Y. (ed), Social structure and personality, New-York, Holt, Rinehard \& Winston, 1961

- DAVIS K.E. \& TODD M.J., Friendship and love relationships, in K.E. Davis (ed), Advances in descriptive psychology, vol.2, Greenwich, CT, JAI Press, 1982

- DEGENNE A., Sur les réseaux de sociabilité, Revue Française de Sociologie, 1983, XXIV, p.109-1 18.

- DERLEGA V.J. \& WINSTEAD B.A. (eds), Friendship and social interaction, New York, SpringerVerlag, 1986

- DICKENS W.J. \& PERLMAN D., Friendship over the life-cycle, in S.W. Duck \& R. Gilmour (eds), Personal relationships, 2, developing personal relationships, New York, Academic Press, 1981

- DOISE W., Les représentations sociales: définition d'un concept, Connexions, $1985, \mathrm{n}^{\circ} 45$

- DUCK S.W. (ed), Handbook of Personal relationships. Theory, research and interventions, New York, John Wiley \& Sons, 1988

- DUCK S.W. (ed), Personal relationships 4: dissolving personal relationships, London, Academic Press, 1982

- DUCK S.W. \& GILMOUR R. (eds), Personal relationships 3: developing personal relationships, London, Academic Press, 1981

- EASTERLING P., Friendship and the Greeks, in R. Porter \& S. Tomaselli (eds), The dialectics of friendship, London, Routledge, 1989

- EISENSTADT S.N., Ritualized personal relations, Man, 1956, nº6, p.90-95

- EISENSTADT S.N., RONIGER L., Patrons, clients and friends; interpersonal relations and the structure of trust in society, Cambridge University Press, 1984, 343 p.

- FELD S.L., The structured use of personal associates, Social Forces, 1984, n62, 3, p.640-652

- FERRAND A., Amis et associés, CESOL, 1985-86, Fasc. 1, 2, 6.

- FERRAND A., Amis et associés, CESOL, 1986, Fasc. 10

- FERRAND A., Une relation interpersonnelle peut-elle être irrévocable?, Colloque de l'AISLF, Genève, 1988

- FERRAND A., Connaissances passagères et vieux amis, les durées de vie des relations interpersonnelles,

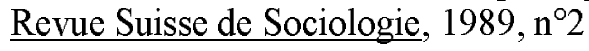


- FERRAND A., For a structural analysis of relational contents, European Conference on Social Network Analysis, Groningen, June 1989

- FERRAND A., L'inverse de l'ordre; essai d'interprétation d'un type d'amitié, n.p., 1990, LASMASCNRS

- FIRTH R., HUBERT J., FORGE A., Families and their relatives, kinship in a middle-class suburb of London, London, Routledge and Kegan, 1969

- FISCHER C.S., To dwell among friends; personal networks in town and city, Chicago, London, The University of Chicago Press, 1982, $451 \mathrm{p}$.

- FLAMENT C., Théorie des graphes et structures sociales, Paris, Gauthier-Villars, Mouton, 1965

- FLAMENT C., Structure et dynamique des représentations sociales, in Jodelet D. (ed), Les représentations sociales, Paris, Presses Universitaires de France, 1989, p.204-219

- FRAISSE J.C., Philia, La notion d'amitié dans la philosophie antique, Paris, Librairie philosophique J. Vrin, 1974

- FURNHAM A., Friendship and personal development, in R. Porter \& S. Tomaselli (eds), The dialectics of friendship, London, Routledge, 1989

- GINSBURG G.P., Rules, scripts and prototypes in personal relationships, in S.W. Duck (ed), Handbook of personal relationships, New York, John Wiley \& Sons, 1988

- GRAFMEYER Y. \& JOSEPH I., L'école de Chicago, naissance de l'écologie urbaine, Paris, Aubier, 1979

- GRANOVETTER M.S., The strength of weak ties, American Journal of Sociology, 1973, p.1361-1380.

- GRANOVETTER M.S., Getting a job, a study of contacts and careers, Harvard University Press, 1974

- GRANOVETTER, M.S., The strength of weak ties; a network theory revisited. In Marsden P., Lin N. (eds), Social structure and network analysis, Beverly Hills, Sage, 1982, p. 105-130.

- HANNERZ U., Explorer la ville, Paris, Ed. de Minuit, 1983

- HAYS R.B., Friendship, in S.W. Duck (ed), Personal relationships, New York, John Wiley \& Sons, 1988

- HERAN F., Comment les Français voisinent, Economie et Statistiques, 1987, n 195, p.43-59

- HERAN F., Les relations de voisinage, Données sociales, INSEE, 1987, p.326-337

- HERAN F., La sociabilité, une pratique culturelle, Economie et statistiques, 1988, n²16, p.3-21

- HERAN F., Mixité et "homolalie"; les rapports entre les sexes dans la vie quotidienne des Français, in Les ménages, mélanges en l'honneur de J. Desabie, INSEE, 1989, p.431-445.

- HERAN F., Trouver à qui parler: le sexe et l'âge de nos interlocuteurs, Données sociales, INSEE, 1990 
- HUCKFELDT R.R., Social contexts, social networks, and urban neighborhoods: environmental constraints on friendship choices, American Journal of Sociology, 1983, Vol 3, p.650-669.

- HUGUES E.C., Men and their work, Glencoe, Free Press, 1958

- LABOV W. Sociolinguistique, Paris, Ed. de Minuit, 1976

- LAGRANGE H. \& ROCHE S., Baby alone in Babylone, CERAT, Institut d'Etudes Politiques de Grenoble, 1988

- LAZARSFELD P.F \& MERTON R.K, Friendship as social process: a substantive and methodological analysis, in Berger M., Abel T. \& Page C. (eds), Freedom and control in modern society, New York, Van Nostrand, 1954

- MAC CALL G.J., The organizational life cycle of relationships, in Duck S.W. (ed), Handbook of personal relationships, New York, John Wiley \& Sons, 1988

- MAISONNEUVE J., Psycho-sociologie des affinités, Paris, Presses Universitaires de France, 1966

- MARSDEN P. \& CAMPBELL K., Measuring ties strength, Social Forces, 1984, nº63, 2, p.482-501

- MARSDEN P. \& LIN N., Social structure and network analysis, Beverly Hills, Sage, 1982

- MORENO J.L., Who shall survive, Washington, Nervous and mental disease publishing Co, 1934

- MORENO J.L., Fondements de la sociométrie, Presses Universitaires de France, 1954

- MOSCOVICI S., La psychanalyse, son image et son public, Paris, PUF, 1976, lè ed. 1961

- ORMEL J., Van TILBURG T.G. \& Van SONDEREN F.L., Personal network delineatin and social support; a comparison of four delineation methods, European Conference on Social Network Analysis, Groningen, June 1989

- PAINE R., In search of friendship. An exploratory analysis in "middle-class" culture, Man, 1969, vol.4, p.505-524

- PAINE R., Anthropological approaches to friendship, Journal of the institute of man, 1970, $\mathrm{n}^{\circ} 1, \mathrm{p} .139$ 159

- PARADEISE C., Sociabilité et culture de classe, Revue Française de Sociologie, 1980, XXI, p.571-597

- PARK R. \& BURGUESS E.W., The city, Chicago, The University of Chicago Press, 1925

- PERLMAN D. \& DUCK S.W. (eds), Intimate relationships: development, dynamics and deterioration, Beverly Hills, Sage, 1987

- PITT-RIVERS J., People of the Sierra, Chicago, Phoenix, 1963

- PITT-RIVERS J., Kinship: pseudo-kinship, in International Encyclopedia of the Social Sciences, New York, MacMillan, Free Press, 1968 
- POGREBIN L.C., Among friends: who we like, why we like them, and what we do with them, New York, McGraw-Hill, 1987

- REICHMAN S., De la menace du chômage à sa réalité: structure du réseau et perception du support social, in "Un niveau intermédiaire: les réseaux sociaux", Actes du séminaire CESOL, Paris, 1987

- ROGERS E.M., Progress, problems and prospects for network research: investigating relationships in the age of electronic communication technologies, Social Networks, $1987, \mathrm{n}^{\circ} 9, \mathrm{p} .285-310$

- SCHUTTE J.G., LIGHT J.M, The relative importance of proximity and status for friendship choices in social hierarchies, Social Psychology, 1978, Vol 41, n³, p.260-264.

- SEMIN G., Prototypes et représentations sociales, in D. Jodelet (ed), Les représentations sociales, Paris, PUF, 1989

- SENNETT R., Les tyrannies de l'intimité, Paris, Le Seuil, 1980 a

- SENNETT R, Familles contre la ville, Paris, Encres-recherches, 1980 b

- SERAFICA F.C., Conceptions of friendship and interaction between friends: an organismicdevelopmental persopective, in F.C. Serafica (ed), Social-cognitive development in context, London, Methuen, 1983

- SILVER A., Friendship and trust as moral ideals: an historical approach, Archives Européennes de Sociologie, Cambridge University Press, 1989, Vol. XXX, p.274-297.

- SIMMEL G., Sociologie et épistémologie, Paris, PUF, 1981

- SIMMEL G., The sociology of G.SIMMEL, Glencoe, Ill., The Free Press, 1950.

- SPERBER D., L'étude anthropologique des représentations: problèmes et perspectives, in D. Jodelet (ed), Les représentations sociales, Paris, PUF, 1989

- SUTTLES G.D., Friendship as a social institution, in G.J. Mc Call (Ed), Social relationships, Chicago, Aldine, 1970

- TELFER E., Friendship, Proceedings of the Aristotelian Society, 1970, LXXI, p.223-241

- VERBRUGGE L.M., The structure of adult friendship choices, Social Forces, 1977, n 56, Vol 2, p.576597.

- WELLMAN B., Applying network analysis to the study of support, in B.H. Gottlieb (ed), $\underline{\text { Social network }}$ and social support, Sage, 1981

- WELLMAN B., Network analysis: some basic principles, in Collins R. (ed), Sociological theory, Washington, Jossey-Bass Publ., 1983

- WELLMAN B., From social support to social network, in I. \& B. Sarason (eds), Social support, The Hague, Martinus Nijhoff, 1985 
- WELLMAN B., Analyse des réseaux sociaux. Principes, développements, productions. in "Un niveau intermédiaire: les réseaux sociaux", Actes du séminaire CESOL, Paris, 1987

- WELLMAN B., Structural analysis: from method and metaphor to theory and substance, in Wellman \& Berkowitz (eds), Social structures: a network approach, Cambridge University Press, 1988

- WILLMOTT P., Friendship networks and social support, London, Policy Studies Institute, 1987

- WIRTH L., Urbanism as a way of life, The American Journal of Sociology, 1938, n 1, p. 1-24

- YOUNG M. \& WILLMOTT P., Le village dans la ville, Paris, Centre de création industrielle, Centre G. Pompidou, 1983 